\title{
The accuracy of prediction of body weight from body measurements in beef cattle ${ }^{*}$
}

\author{
SERKAN OZKAYA ${ }^{1}$ and YALCIN BOZKURT ${ }^{1}$ \\ Department of Animal Science, Faculty of Agriculture, Süleyman Demirel University, Isparta, Turkey
}

\begin{abstract}
The objective of this study was to determine the accuracy of prediction of body weight from body measurements in beef cattle. Wither height, chest girth, body length, chest depth, hip width and hip height measurements were obtained from Holstein, Brown Swiss and crossbred $(n=140)$. Determination coefficients $\left(R^{2}\right)$ of regression equation that included all body measurements were higher in Brown Swiss and crossbred than Holstein (92.2, 95.0 and $68.2 \%$, respectively). However, it was found that chest girth was the best parameter of all for prediction of body weight in Brown Swiss $\left(R^{2}=91.1 \%\right)$ and crossbred cattle $\left(R^{2}=88.8 \%\right)$ in comparison to Holstein $\left(R^{2}=60.7 \%\right)$. According to these results, the body weight estimation of Brown Swiss and crossbred cattle using the body measurements produced higher prediction accuracies than Holstein but chest girth was the best parameter to prediction of body weight among all body measurements. However, the prediction accuracy of prediction of body weight from body measurements and also chest girth was decreased when the animals frame size was increased.
\end{abstract}

Keywords: cattle, body measurements, body weight, Holstein, Brown Swiss, crossbred

\section{Zusammenfassung}

\section{Die Genauigkeit einer Vorhersage des Körpergewichtes mittels Körpermaßen bei Schlachtrindern}

Ziel vorliegender Studie war die Bestimmung der Genauigkeit der Vorhersage des Körpergewichtes von Schlachtrindern mit Hilfe von Körpermaßen. Bei 140 Schlachtrindern der Rassen Holstein und Brown Swiss sowie deren Kreuzungen wurden folgende Körpermaße erfasst: Widerristhöhe (wither height), Brustumfang (chest girth), Körperlänge (body length), Brusttiefe (chest depth), Hüftbreite (hip width) und Hüfthöhe (hip height). Die Regressionskoeffizienten lagen bei den Brown Swiss und Kreuzungstieren höher als bei Holstein Rindern. Für die Vorhersage des Körpergewichtes erwies sich der Brustumfang mit $R^{2}=91,1 \%$ bei Braun Swiss, bei den Kreuzungstieren mit $R^{2}=88,8 \%$ und bei den Holstein mit $R^{2}=60,7 \%$ als bester Parameter. Allerdings verringert sich bei Nutzung des Brustumfanges die Genauigkeit der Vorhersage mit zunehmender Rahmengröße der Tiere.

Schlüsselwörter: Rinder, Körpermaße, Körpergewicht, Holstein, Brown Swiss, Kreuzungstiere

\footnotetext{
* The study was presented at 5th National Animal Science Congress in Van/Turkiye and the abstract was published in book of abstracts.
} 


\section{Introduction}

Body weight of animals is an important factor associated with several management practices including selection for slaughter or breeding, determining feeding levels and also it is a good indicator of animal condition (ULUTAS et al. 2001).

The relationship between body measurements and body weight depends upon breed, age, type, size, condition and fattening level of the animals (HEINRICHS et al. 1992, YANAR et al. 1995). VAN MARLE-KÖSTER et al. (2000) described body measurements as selection criteria for growth in cattle.

HEINRICHS et al. (1992) indicated that body measurements can be used for prediction of body weight. GILBERT et al. (1993) reported that there is close correlation between body weight and body measurements. MSANGl et al. (1999), SLIPPERS et al. (2000), FOUIRE et al. (2002), WILLEKE and DÜRSCH (2002) and BOZKURT (2006) indicated that chest girth can be used to predict body weight that it is the best prediction parameter. CAGLAR and SEKERDEN (1993) declared that the regression equations must be determined for all beef breeds for different country and region.

The aim of this study was to determine the accuracy of prediction of body weight from metric body measurements in beef cattle.

\section{Material and methods}

This study were carried out in Isparta and Burdur provinces in the Mediterranean part of Turkey and 140 male animals in total were used and comprised of 56 Holstein (body weight ranging 337 to $677 \mathrm{~kg}$ ), 30 Brown Swiss (body weight ranging 326 to $930 \mathrm{~kg}$ ) and 54 Crossbred cattle (body weight ranging 326 to $677 \mathrm{~kg}$ ).

Body weight of animals was determined by using a digital weighing scale prior to slaughter (Marmara $0580 \mathrm{MEB}$ ). The parameters such as body weight, chest girth, wither height, body length, chest depth, hip width and hip height were measured using measuring stick and tape (Hauptner, Germany) when animals were standing as described in OZKAYA and BOZKURT (2008).

The best prediction equations for body weight from other traits (chest girth, body length, wither height, chest depth, hip width and hip height) as independent variables were determined. Descriptive statistics and regression analysis of body weight on each of the independent variables were performed using the MINITAB, 13 Inc (2001). Comparisons between means were determined by Tukey test.

Correlation coefficients were also obtained from parameters. Linear, quadratic and cubic effects of independent variables on body weight were included in the following model:

$$
Y_{i}=b_{0}+b_{1} x_{i}+b_{2} x_{i}^{2}+b_{3} x_{i}^{3}+e_{i}
$$

where is $Y_{i}$ the body weight observation of an $i$-th animal, $b_{0}$ the intercept, $b_{1}, b_{2}, b_{3}$ the corresponding linear, quadratic and cubic regression coefficients, $x_{i}$ the body measurement (chest girth, body length, wither height, chest depth, hip width and hip height) and $e_{i}$ the residual error term. 


\section{Results and discussion}

Descriptive statistics of body weight and body traits are shown in Table 1. The parameters of Holstein were higher and statistically significant $(P<0.05)$ than Brown Swiss and crossbreds. All parameters were found no significant between Brown Swiss and crossbred cattle but only wither height values were found statistically significant in all breeds $(P<0.05)$.

Table 1

Descriptive statistics for body weight and body measurements

Beschreibende Statistik für Körpergewicht und Körpermaße

\begin{tabular}{|c|c|c|c|}
\hline Variables & Breeds & $n$ & Mean \pm SE \\
\hline Body weight, kg & $\begin{array}{l}\text { Holstein } \\
\text { Brown Swiss } \\
\text { Crossbred }\end{array}$ & $\begin{array}{l}30 \\
56 \\
54\end{array}$ & $\begin{array}{ll}513.4^{\mathrm{a}} & \pm 11.6 \\
440.7^{\mathrm{b}} & \pm 25.7 \\
460.9^{\mathrm{b}} & \pm 13.6\end{array}$ \\
\hline Body length, $\mathrm{cm}$ & $\begin{array}{l}\text { Holstein } \\
\text { Brown Swiss } \\
\text { Crossbred }\end{array}$ & $\begin{array}{l}30 \\
56 \\
54\end{array}$ & $\begin{array}{lll}146.37^{\mathrm{a}} & \pm 0.95 \\
136.88^{\mathrm{b}} & \pm 1.97 \\
140.15^{\mathrm{b}} & \pm 1.26\end{array}$ \\
\hline Wither height, $\mathrm{cm}$ & $\begin{array}{l}\text { Holstein } \\
\text { Brown Swiss } \\
\text { Crossbred }\end{array}$ & $\begin{array}{l}30 \\
56 \\
54\end{array}$ & $\begin{array}{lll}132.60^{\mathrm{a}} & \pm 0.66 \\
123.45^{\mathrm{b}} & \pm 1.40 \\
127.95^{\mathrm{c}} & \pm 1.14\end{array}$ \\
\hline Hip height, $\mathrm{cm}$ & $\begin{array}{l}\text { Brown Swiss } \\
\text { Holstein } \\
\text { Crossbred }\end{array}$ & $\begin{array}{l}30 \\
56 \\
54\end{array}$ & $\begin{array}{lll}137.20^{\mathrm{a}} & \pm 0.68 \\
128.87^{\mathrm{b}} & \pm 1.46 \\
132.16^{\mathrm{b}} & \pm 1.12\end{array}$ \\
\hline Hip width, $\mathrm{cm}$ & $\begin{array}{l}\text { Holstein } \\
\text { Brown Swiss } \\
\text { Crossbred }\end{array}$ & $\begin{array}{l}30 \\
56 \\
54\end{array}$ & $\begin{array}{l}46.152^{\mathrm{a}} \pm 0.58 \\
43.283^{\mathrm{b}} \pm 0.87 \\
43.222^{\mathrm{b}} \pm 0.56\end{array}$ \\
\hline Chest depth, $\mathrm{cm}$ & $\begin{array}{l}\text { Holstein } \\
\text { Brown Swiss } \\
\text { Crossbred }\end{array}$ & $\begin{array}{l}30 \\
56 \\
54\end{array}$ & $\begin{array}{l}68.488^{b} \pm 0.75 \\
64.78^{b} \pm 1.12 \\
65.074^{b} \pm 0.68\end{array}$ \\
\hline Chest girth, cm & $\begin{array}{l}\text { Holstein } \\
\text { Brown Swiss } \\
\text { Crossbred }\end{array}$ & $\begin{array}{l}30 \\
56 \\
54 \\
\end{array}$ & $\begin{array}{lll}189.36^{\mathrm{a}} & \pm 1.73 \\
180.25^{\mathrm{b}} & \pm 3.38 \\
181.59^{\mathrm{b}} & \pm 1.66 \\
\end{array}$ \\
\hline
\end{tabular}

$\overline{a, b, c}$ means in a column bearing different superscript are significantly $(P<0.05)$ different

The best regression equations of body weight on various body measurements are shown in Table 2. Results of regressions of body weight on the linear, quadratic and cubic effects of each body measurements are presented in Table 3.

Chest girth was statistically significant for all breeds (Table 2). The $R^{2}$ value for Holstein was obtained from the equation contained body length, wither height, chest depth and chest girth was found $66.1 \%$. This result was lower than findings of TUZEMEN et al. (1995) who reported $R^{2}=90.7 \%$. The $R^{2}$ value obtained from the equation contained body length, wither height and chest girth was found $66.1 \%$ and this result was lower than findings of SEKERDEN et al. (1991) $\left(R^{2}=97.7 \%\right) . R^{2}$ value was obtained from the equation contained only chest girth $\left(R^{2}=60.7 \%\right)$ was lower than those findings of SEKERDEN et al. (1991) $\left(R^{2}=97.3 \%\right)$ and TUZEMEN et al. (1995) $\left(R^{2}=86.7 \%\right)$.

The highest $R^{2}$ value for Brown Swiss were obtained from the equation contained all body measurements $\left(R^{2}=92.2 \%\right)$ (Table 2$) . R^{2}$ value was determined as $91.8 \%$ which contained body length, wither height, chest depth and chest girth. This result was in line 
with findings of TUZEMEN et al. (1993) $\left(R^{2}=90.7 \%\right)$ and BOZKURT (2006) $\left(R^{2}=93.6 \%\right)$. $R^{2}$ value was obtained from the equation contained only chest girth was higher than those findings of TUZEMEN et al. (1993) and BOZKURT (2006) $\left(R^{2}=91.1,86.9\right.$ and 89.9\%, respectively).

Table 2

The best prediction equations of body weight

Beste Vorhersagegleichungen des Körpergewichtes

\begin{tabular}{|c|c|c|}
\hline Breeds & Regression equations & $R^{2}, \%$ \\
\hline \multirow{6}{*}{ Holstein } & $Y=-784+3.56 \mathrm{BL}^{*}+7.48 \mathrm{WL}-6.79 \mathrm{HH}-0.77 \mathrm{HW}-0.45 \mathrm{CD}+4.14 \mathrm{CG}^{*}$ & 68.2 \\
\hline & $Y=-872+2.91 \mathrm{BL}+2.19 \mathrm{WH}-0.22 \mathrm{BD}+3.62 \mathrm{CG}^{*}$ & 66.1 \\
\hline & $Y=867+2.87 \mathrm{BL}+2.11 \mathrm{WH}+3.59 \mathrm{CG}^{*}$ & 66.1 \\
\hline & $Y=-473+5.21 C^{*}$ & 60.7 \\
\hline & $Y=-715+8.39 \mathrm{BL}^{*}$ & 47.7 \\
\hline & $Y=-1006+11.5 \mathrm{WH}^{*}$ & 43.2 \\
\hline \multirow{6}{*}{ Brown Swiss } & $Y=-868+2.28 \mathrm{BL}-0.10 \mathrm{CWH}-0.94 \mathrm{HH}+4.03 \mathrm{HW}-4.35 \mathrm{CD}+6.87 \mathrm{CG}^{*}$ & 92.2 \\
\hline & $Y=-883+2.53 \mathrm{BL}-0.69 \mathrm{WH}-5.30 \mathrm{BD}+7.81 \mathrm{CG}^{*}$ & 91.8 \\
\hline & $Y=-866+1.36 \mathrm{BL}-1.00 \mathrm{WH}+6.91 \mathrm{CG}^{*}$ & 91.3 \\
\hline & $Y=-869+7.27 C^{*}$ & 91.1 \\
\hline & $Y=-1156+11.7 \mathrm{BL}^{*}$ & 79.2 \\
\hline & $Y=-1466+15.4 \mathrm{WH}^{*}$ & 70.8 \\
\hline \multirow{5}{*}{ Crossbred } & $Y=-1065+1.76 \mathrm{BL}-1.27 \mathrm{WH}+3.48 \mathrm{HH}^{*}+2.63 \mathrm{HW}+0.81 \mathrm{CD}+4.49 \mathrm{CG}^{*}$ & 95.0 \\
\hline & $Y=-1068+2.80 \mathrm{BL}^{*}+1.76 \mathrm{WH}+5.02 \mathrm{CG}^{*}$ & 93.9 \\
\hline & $Y=-935+7.69 C^{*}$ & 88.8 \\
\hline & $Y=-912+9.80 \mathrm{BL}^{*}$ & 82.2 \\
\hline & $Y=-836+10.1 \mathrm{WH}^{*}$ & 71.8 \\
\hline
\end{tabular}

BW body weight, BL body length, WH wither height, HH hip height, HW hip width, CD chest depth, CG chest girth, * ${ }^{*}$ statistically significant $(P<0.05)$

The highest $R^{2}$ value for crossbred was obtained from equation contained all body measurements $\left(R^{2}=95.0 \%\right)$. In addition, $R^{2}$ value was found $88.8 \%$ which included only CG (Table 2).

The highest $R^{2}$ value was obtained from chest girth for all breeds (Table 3). For Holstein, $R^{2}$ was found $61.5 \%$ and this result was lower than those findings of HEINRICHS et al. (1992) and WILSON et al. (1997) $\left(R^{2}=95\right.$ and $97 \%$, respectively). In the present study, results showed that when the body weight increased to $500 \mathrm{~kg}$, the prediction accuracy of body weight from chest girth was decreased for Holstein $\left(R^{2}=39.4 \%\right)$.

Linear, quadratic and cubic coefficients of chest girth for Brown Swiss were found 91.1, 94.3 and $94.4 \%$, respectively (Table 3 ). These results were higher than findings of BOZKURT (2006) (89.9, 60.1 and $90.2 \%$, respectively).

The highest $R^{2}$ value was found in body length and chest girth for crossbreds (Table 3 ) $\left(R^{2}=82.2\right.$ and $88.8 \%$, respectively). The cubic term was statistically significant for body length and chest girth $(P<0.05)$.

The correlation coefficients of traits are shown in Table 4.

The highest correlation was obtained between body weight and chest girth. The correlation coefficient $(r=0.78)$ between body weight and chest girth for Holstein was lower than those findings of SEKERDEN et al. (1991) and TUZEMEN et al. (1995) ( $r=0.99$ and 0.83, respectively). For Brown Swiss, correlation coefficient between body weight and 
Table 3

Regressions of body weight on linear, quadratic and cubic effects of each body measurements

Effekte linearer, quadratischer und kubischer Regressionsmodelle bei einzelnen Körpermaßen zum Körpergewicht

\begin{tabular}{|c|c|c|c|c|c|c|}
\hline Breeds & Variables & Intercept & Linear & Quadratic & Cubic & $R^{2}, \%$ \\
\hline \multirow{18}{*}{ Holstein } & \multirow{3}{*}{ body length } & -714.76 & 8.39 & - & - & 47.7 \\
\hline & & -1680.75 & 21.43 & $-0.04^{\mathrm{ns}}$ & - & 47.9 \\
\hline & & -32530.30 & 642.95 & $-4.21^{\mathrm{ns}}$ & $0.009^{\text {ns }}$ & 48.4 \\
\hline & \multirow[t]{3}{*}{ wither height } & -1006.16 & 11.46 & - & - & 43.2 \\
\hline & & -3922.73 & 55.72 & $-0.16^{\mathrm{ns}}$ & - & 43.6 \\
\hline & & 7538.90 & -205.39 & $1.81^{\mathrm{ns}}$ & $-0.005^{\mathrm{ns}}$ & 43.6 \\
\hline & \multirow[t]{3}{*}{ hip height } & -938.10 & 10.58 & - & - & 39.2 \\
\hline & & -5661.64 & 79.62 & $-0.25^{\mathrm{ns}}$ & - & 39.9 \\
\hline & & -178552.00 & 3857.90 & $-27.76^{\mathrm{ns}}$ & $0.07^{\mathrm{ns}}$ & 41.6 \\
\hline & \multirow[t]{3}{*}{ hip width } & 132.56 & 8.25 & - & - & 17.3 \\
\hline & & -1906.51 & 88.66 & -0.78 & - & 41.5 \\
\hline & & -5475.86 & 301.64 & -4.93 & $0.026^{\mathrm{ns}}$ & 42.4 \\
\hline & \multirow[t]{3}{*}{ chest depth } & -63.85 & 8.43 & - & - & 30.2 \\
\hline & & 1662.49 & -43.98 & 0.39 & - & 39.2 \\
\hline & & 4587.18 & -184.99 & 2.62 & $-0.012^{\mathrm{ns}}$ & 40.0 \\
\hline & \multirow[t]{3}{*}{ chest girth } & -473.22 & 5.21 & - & - & 60.7 \\
\hline & & 740.12 & -7.84 & $0.03^{\text {ns }}$ & - & 61.5 \\
\hline & & -701.95 & 15.33 & $-0.09^{\text {ns }}$ & $0.0002^{\text {ns }}$ & 61.5 \\
\hline \multirow{18}{*}{ Brown Swiss } & \multirow[t]{3}{*}{ body length } & -1155.63 & 11.66 & - & - & 79.7 \\
\hline & & -463.51 & -10.74 & $0.07^{\mathrm{ns}}$ & - & 80.2 \\
\hline & & 16948.20 & -358.35 & $2.51^{\mathrm{ns}}$ & $-0.005^{\mathrm{ns}}$ & 80.5 \\
\hline & \multirow[t]{3}{*}{ wither height } & -1465.84 & 15.44 & - & - & 70.8 \\
\hline & & -10831.20 & -179.34 & 0.77 & - & 85.2 \\
\hline & & -17734.20 & 508.19 & -4.73 & $-0.015^{\mathrm{ns}}$ & 85.7 \\
\hline & \multirow[t]{2}{*}{ hip height } & -1506.26 & 15.11 & - & - & 73.9 \\
\hline & & 5812.70 & -94.95 & 0.41 & - & 78.1 \\
\hline & & 48390.60 & -1061.28 & $7.70^{\text {ns }}$ & $-0.018^{\mathrm{ns}}$ & 78.5 \\
\hline & \multirow[t]{3}{*}{ hip width } & -683.35 & 25.97 & - & - & 77.9 \\
\hline & & 1743.80 & -80.51 & 1.15 & - & 83.5 \\
\hline & & 10125.40 & -620.78 & 12.64 & $-0.081^{\mathrm{ns}}$ & 84.3 \\
\hline & \multirow[t]{3}{*}{ chest depth } & -859.55 & 20.07 & - & - & 76.2 \\
\hline & & 1800.59 & -57.70 & $0.56^{\mathrm{ns}}$ & - & 79.3 \\
\hline & & 1415.76 & -40.69 & $0.31^{\mathrm{ns}}$ & $0.001^{\text {ns }}$ & 79.3 \\
\hline & \multirow[t]{3}{*}{ chest girth } & -868.79 & 7.26 & - & - & 91.1 \\
\hline & & 1733.22 & -19.84 & 0.07 & - & 94.3 \\
\hline & & -2235.27 & 41.80 & -0.25 & $0.000^{\text {ns }}$ & 94.4 \\
\hline \multirow{18}{*}{ Crossbreds } & \multirow[t]{3}{*}{ body length } & -912.40 & 9.79 & - & - & 82.2 \\
\hline & & 1325.80 & -22.07 & $0.11^{\mathrm{ns}}$ & - & 83.3 \\
\hline & & 55551.10 & -1180.85 & $8.34^{\mathrm{ns}}$ & -0.019 & 86.6 \\
\hline & \multirow{3}{*}{ wither height } & -835.90 & 10.14 & - & - & 71.8 \\
\hline & & -1012.60 & 12.86 & $-0.011^{\mathrm{ns}}$ & - & 71.8 \\
\hline & & 30886.90 & -724.36 & $5.653^{\mathrm{ns}}$ & $-0.014^{\mathrm{ns}}$ & 72.5 \\
\hline & \multirow[t]{2}{*}{ hip height } & -934.20 & 10.57 & - & - & 75.2 \\
\hline & & -426.60 & 2.91 & $0.029^{\text {ns }}$ & - & 75.3 \\
\hline & & 55569.50 & -1255.93 & $9.436^{\text {ns }}$ & -0.023 & 77.7 \\
\hline & hip width & -428.90 & 20.59 & - & - & 72.9 \\
\hline & & 2073.50 & -97.67 & 1.38 & - & 79.9 \\
\hline & & 1916.40 & -86.20 & 1.10 & $0.002^{\mathrm{ns}}$ & 79.9 \\
\hline & chest depth & -582.10 & 16.03 & - & - & 63.7 \\
\hline & & 1550.10 & -49.30 & $0.50^{\text {ns }}$ & - & 66.0 \\
\hline & & 22920.30 & -1042.37 & $15.81^{\mathrm{ns}}$ & $-0.078^{\mathrm{ns}}$ & 67.9 \\
\hline & chest girth & -935.10 & 7.69 & - & - & 88.8 \\
\hline & & 1131.20 & -14.96 & $0.06^{\mathrm{ns}}$ & - & 89.5 \\
\hline & & 13901.30 & -226.40 & $1.22^{\mathrm{ns}}$ & $-0.002^{\mathrm{ns}}$ & 89.8 \\
\hline
\end{tabular}

ns statistically non significant $(P>0.05)$ 
chest girth was found 0.95. These result was in line with findings of BOZKURT (2006) but was higher than YANAR et al. (1995) ( $r=0.86)$. For crossbreds, the correlation coefficient between body weight and chest girth was similar with Brown Swiss but higher than Holstein (Table 4).

In conclusion, this study showed that prediction accuracy of body weight using metric body measurements in Brown Swiss and crossbred was higher than Holstein. However, the prediction accuracy of chest girth was higher than other traits for prediction of body weight. However, prediction accuracy of body weight using metric body measurements was decreased in big size animals.

Table 4

Correlation coefficients between body weight and body measurements

Korrelationskoeffizienten zwischen Körpergewicht und Körpermaßen

\begin{tabular}{lccc}
\hline Variables & Holstein & $\begin{array}{c}\text { Breeds } \\
\text { Brown Swiss }\end{array}$ & Crossbred \\
\hline Body length & 0.69 & 0.89 & 0.91 \\
Wither height & 0.66 & 0.84 & 0.85 \\
Hip height & 0.63 & 0.86 & 0.87 \\
Hip width & 0.61 & 0.88 & 0.85 \\
Chest depth & 0.64 & 0.87 & 0.80 \\
Chest girth & 0.78 & 0.95 & 0.94 \\
\hline
\end{tabular}

\section{Acknowledgements}

We would like to thank the Scientific Research Unit of Süleyman Demirel University for funding this study. Project no. 1047-YL-05

\section{References}

Bozkurt Y (2006) Prediction of body weight from body size measurements in Brown Swiss feedlot cattle fed under small-scale farming conditions. J Appl Anim Res 29, 29-32

Caglar H, Sekerden $\mathrm{O}$ (1993) Predicting live weight from various body measurements in Yerli Kara cattle. J Agri Collage of Ondokuzmayıs University 8, 45-7

Fouire PJ, Neser FWC, Oliver JJ, Van der Westhizen C (2002) Relationship between production performance, visual appraisal and body measurements of young Dorper rams. South African J Anim Sci 32, 256-62

Gilbert RP, Bailey DRC, Shannon NH (1993) Linear body measurements of cattle before and after 20 years of selection for postweaning gain when fed two different diets. J Anim Sci 71, 1712-20

Heinrichs AJ, Rogers GW, Cooper JB (1992) Predicting body weight and wither height in Holstein heifers using body measurements. J Dairy Sci 75, 3576-81

Minitab Institute Inc. (2001) MINITAB User's Guide. Release 13 for Windows. MINITAB Inc. State Collage, PA, USA

Msangi BSJ, Bryant MJ, Kavana Y, Msanga N, Kizima JB (1999) Body measurements as a management tool far crossbred dairy cattle at a smallholder farm Ksition. Proced Tanzania Soc Anim Sci 26, 168-75

Ozkaya S, Bozkurt Y (2008) The relationship of parameters of body measures and body weight by using digital image analysis in pre-slaughter cattle. Arch Tierz 51, 120-8

Sekerden O, Kuran M, Sen SO (1991) Predicting live weight from various body measurements in BlackWhite cattle. Turkish J Vet Anim Sci 16, 93-102

Slippers SC, Letty BA, De Villerrs JF (2000) Prediction of body weight of Nguni goats. South African J Anim Sci 30 Suppl 1, 127-8 
Tuzemen N, Yanar M, Akbulut O, Ockerman HW (1993) Prediction of body weights from body measurements in Brown swiss calves reared in Eastern region of Turkey. World Rev Anim Prod 28, no. 4, Oct-Dec.

Tuzemen N, Yanar M, Akbulut O, Ugur F, Aydin R (1995) Prediction of body weights from body measurements in Holstein calves. J Agri Collage of Ataturk University 26, 245-52

Ulutas Z, Saatci M, Ozluturk A (2001) Prediction of body weights from body measurements in East Anatolian Red Calves. J Agri Collage of Ataturk University 32, 61-5

Van Marle-Köster E, Mostert BE, Van der Westhuizen J (2000) Body measurements as selection criteria for growth in South African Hereford cattle. Arch Tierz 43, 5-15

Yanar M, Tuzemen N, Ozkan M, Aydin R, Ugur F (1995) Prediction of body weights from body measurements in Brown Swiss cattle. Turkish J Vet Anim Sci 19, 357-60

Willeke H, Dürsch T (2002) Prediction of the body weight of Simmental heifers using heart girth measurements. Arch Tierz 45, 23-8 [in German]

Wilson LL, Egan CL, Terosky TL (1997) Body measurement and body weight of special-fed Holstein veal calves. J Dairy Sci 30, 3077-82

Received 24 October 2008, accepted 24 March 2009.

Corresponding author:

SERKAN OZKAYA, MSC

email: sozkaya@ziraat.sdu.edu.tr

Department of Animal Science, Faculty of Agriculture, Süleyman Demirel University, 32260 Cunur-Isparta, Turkey 